\title{
A review of the role of the gut microflora in irritable bowel syndrome and the effects of probiotics
}

\author{
J. A. J. Madden and J. O. Hunter* \\ Gastroenterology Research Unit, Unit E7, Box 201 A, Addenbrookes NHS Trust, Hill's Road, Cambridge CB2 2QQ, UK
}

\begin{abstract}
Irritable bowel syndrome (IBS) is a multi-factorial gastrointestinal condition affecting $8-22 \%$ of the population with a higher prevalence in women and accounting for $20-50 \%$ of referrals to gastroenterology clinics. It is characterised by abdominal pain, excessive flatus, variable bowel habit and abdominal bloating for which there is no evidence of detectable organic disease. Suggested aetiologies include gut motility and psychological disorders, psychophysiological phenomena and colonic malfermentation. The faecal microflora in IBS has been shown to be abnormal with higher numbers of facultative organisms and low numbers of lactobacilli and bifidobacteria. Although there is no evidence of food allergy in IBS, food intolerance has been identified and exclusion diets are beneficial to many IBS patients. Food intolerance may be due to abnormal fermentation of food residues in the colon, as a result of disruption of the normal flora. The role of probiotics in IBS has not been clearly defined. Some studies have shown improvements in pain and flatulence in response to probiotic administration, whilst others have shown no symptomatic improvement. It is possible that the future role of probiotics in IBS will lie in prevention, rather than cure.
\end{abstract}

Gut microflora: Irritable bowel syndrome: Probiotics: Fermentation

\section{Introduction}

Irritable bowel syndrome (IBS) is a poorly understood gastrointestinal (GI) condition that typically begins in early adult life (Maxwell et al. 1997). It is believed to affect approximately one-fifth of the population, though it is estimated that $60-75 \%$ of symptomatic people do not seek medical attention in the UK (Farthing, 1995). Typical symptoms include abdominal pain, excessive flatus and variable bowel habit for which no endoscopic, radiological, histological, biochemical or microbiological cause is apparent. The lack of positive tests makes the diagnosis of IBS one of exclusion (Maxwell et al. 1997). The Modified Rome Criteria (Thompson et al. 1999; Table 1) provide a means of standardisation of patients with IBS recruited to research studies, but do not allow a specific diagnosis.

The cause of IBS is not yet known. Suggestions include psychosocial factors, altered GI motility, heightened sensory function of the intestine, or malfermentation of food residues (Hunter, 1991; Camilleri, 2001). It may be that IBS is, in reality, a group of separate conditions producing similar symptoms.

Interest in fermentation arose from the suggestion that disruption of the intestinal microflora may be important in the pathogenesis of IBS. Gastroenteritis, surgery and antibiotics are all known to alter the microflora as are several drug classes, including antineoplastic drugs, immunosuppressive agents and histamine $\mathrm{H}_{2}$ antagonists (Hooker \& dePiro, 1988; Neilson et al. 1994). Over $40 \%$ of patients questioned in a retrospective study attributed the onset of their symptoms to a definite event, such as a course of antibiotics, abdominal or pelvic surgery, or a bout of gastroenteritis (Hunter \& Alun Jones, 1985).

The role of bacterial gastroenteritis in the onset of IBS symptoms has been extensively studied. Gwee et al. (1996) provided questionnaires to a group of seventy patients admitted to hospital with acute gastroenteritis. Twenty-two of these patients later developed symptoms compatible with IBS and, of these, twenty still had persistent symptoms after six months. Similarly, Neal et al. (1997) investigated a cohort of 544 people with a laboratory-confirmed diagnosis of bacterial gastroenteritis. Questionnaires were sent to the patients relating to their bowel habit prior to, and after, their episode of gastroenteritis. The Modified Rome Criteria were used to assess the questionnaires for IBS symptoms. Twenty-five per cent of subjects reported persistence of altered bowel habit after six months, with one in fourteen developing symptoms consistent with IBS. The risk of developing IBS was increased in women and those in whom the gastroenteritis caused diarrhoea of longer duration.

In a similar study, Rodríguez \& Ruigómez

(1999)

\footnotetext{
Abbreviations: GI, gastrointestinal; IBS, irritable bowel syndrome.

* Corresponding author: Dr J. O. Hunter, fax +44 1223 211443, email john.hunter@addenbrookes.nhs.uk
} 
Table 1. The Modified Rome Criteria for IBS (Thompson et al. 1999)

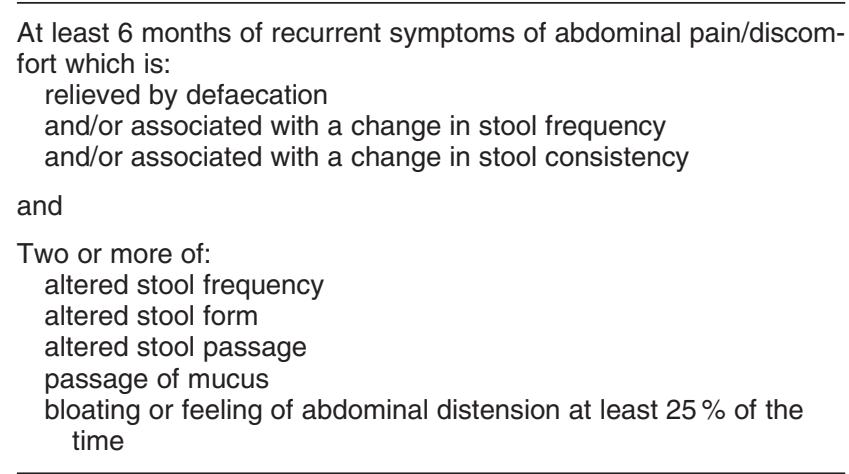

investigated the risk of IBS after bacterial gastroenteritis. They examined a group of patients with a first episode of bacterially confirmed gastroenteritis and compared them with a large control cohort of patients obtained from the General Practice Research Database. Those with a previous history of IBS, cancer or alcoholism were excluded. A follow-up after one year showed that, of the 575169 people in the control group, 2027 developed IBS (incidence/1000 persons years 3.5). In contrast, twelve of the 303 gastroenteritis patients developed IBS (incidence/ 1000 persons years 39.7; relative risk 11.9). These data provide strong evidence that at least one form of IBS may be caused by bacterial infections. Such is the evidence supporting the role of bacterial infection in the aetiology of IBS that Gwee (2001) suggested that the term post-infectious irritable bowel syndrome should be used in patients who present with IBS-type symptoms following a recent confirmed or presumed exposure to infectious organisms, or those who have recently returned from a tropical or developing country.

Antibiotics have also been implicated in the pathogenesis of IBS. Antibiotics are one of the most likely (instead of potential) causes of disruption of the normal GI microflora and are widely used in both human and veterinary medicine. The composition of the GI microflora of man is known to be relatively stable in normal conditions, so that certain bacterial species can be consistently detected in samples collected from the GI tract (Savage, 1977). The stability of the normal human GI microflora is the consequence of several factors including gastric acidity, gut motility, bile salts, immunological defence factors, colonic $\mathrm{pH}$ and competition between micro-organisms for nutrients and intestinal binding sites (Marshall, 1999). These together provide a barrier to disruption of the flora that is known as colonisation resistance (Van der Waiij, 1983). Antibiotics are unarguably important for the treatment and prophylaxis of disease (Lidbeck \& Nord, 1994) but it has been demonstrated both in humans and animals that they can detrimentally affect the ecological balance of the GI microbiota by affecting the indigenous bacterial populations as well as the target population (Lidbeck \& Nord, 1993; Witsell et al. 1995). The indigenous flora takes part in many physiological and pathophysiological reactions and may influence the metabolic activities of certain drugs. All these activities can be affected by antibiotics (Finegold et al. 1983). Antibiotics are sometimes valuable in the treatment of IBS, supporting suggestions that the indigenous microflora may play an important role (Pimental et al. 2000).

The possible role of antibiotics in the aetiology of IBS has been investigated in two prospective studies. Alun Jones et al. (1984) initiated a prospective, double-blind controlled study involving 300 patients undergoing hysterectomy who were administered either prophylactic metronidazole or a placebo. They found a greater incidence of IBS-type symptoms following antibiotic prophylaxis than those receiving the placebo and postulated that a form of IBS exists which follows antibiotic administration. Mendall \& Kumar (1998) investigated 421 subjects attending a general practice clinic. Using the Manning Criteria, forty-eight subjects screened had symptoms of IBS and this was strongly associated with the use of antibiotics (odds ratio (OR) 3.7; $95 \%$ CI 1.80, 7.60).

The risk of developing IBS following a course of analgesics (paracetamol, aspirin, or non-aspirin anti-inflammatory drugs) was also examined in one study. A self-reporting questionnaire was sent to 892 eligible subjects, of whom 643 responded. Of the responders, $12 \%$ reported symptoms related to IBS and the presence of IBS was significantly associated with the use of analgesics (adjusted OR 4.25; $95 \%$ CI $1.36,13.31)$ It was suggested, however, that the use of analgesics was to relieve somatic pains associated with IBS, rather than that analgesics were important in the pathogenesis of IBS (Locke et al. 2000).

\section{The intestinal microflora in irritable bowel syndrome}

There is considerable evidence to show that factors that disturb the gut microflora may contribute to the development of IBS. It seems that such damage to the flora may become permanent. The intestinal microflora in IBS patients has been studied extensively by conventional microbiological techniques. Balsari et al. (1982) investigated the faecal flora of twenty patients with IBS. The faecal flora of IBS patients had significantly lower numbers of coliforms compared with controls and also significantly lower numbers of both lactobacilli and bifidobacteria. They concluded that, although the faecal microflora of patients with IBS was qualitatively very similar to healthy individuals, there were considerable quantitative differences in some of the bacterial species.

Bradley et al. (1987) examined in detail the faecal flora of a patient who suffered from food-related IBS. They found a considerable variation in total bacterial counts over an 18-month period ranging between $1.3 \times 10^{10}$ and $5.9 \times 10^{11} \mathrm{cfu} / \mathrm{g}$ dry weight with a high proportion of facultative organisms, dominated by Streptococcus spp., Escherichia coli and Proteus spp. The dominant anaerobic organisms were Clostridium spp. rather than the usual Bacteroides spp. or Bifidobacterium spp.

Wyatt et al. (1988) examined the faecal microflora of two patients with food-related IBS before and after challenge with foods known to provoke symptoms. There was little change in the major bacterial species during the food challenges, though in one patient, levels of bifidobacteria 
and lactobacilli increased from $21 \%$ to $43 \%$ of the total flora.

The caecal biopsy-associated, caecal luminal and faecal microflora were investigated in six IBS patients fulfilling the Modified Rome Criteria and six controls. Patients received a single $100 \mathrm{ml}$ phosphate enema, rather than full bowel preparation, to clear the left side of the bowel leaving the right side, including the caecum, undisturbed. Carbon dioxide rather than oxygen was used to insufflate the bowel, to maintain anaerobic conditions. There were significantly higher numbers of anaerobes in the stools of healthy subjects compared with IBS patients. In IBS patients, lactobacilli were present in the caecal mucosae and caecal lumen, but were not detectable in the faeces. Aerobes were detected in the caecal mucosae of five IBS patients compared with two of the healthy subjects (Madden et al. 2001).

Thus there is evidence that the intestinal microflora of patients with IBS differs from that of healthy individuals. However, it is not yet possible to be certain whether the changes in the intestinal microflora seen in IBS patients are the cause of IBS, or are merely a result of the disturbed gut motility that IBS causes. More studies are desirable to elucidate this issue.

It is possible that the differences in the gut microflora of patients with IBS produce abnormal colonic fermentation. Fermentation can be defined as the anaerobic breakdown of carbohydrate and protein by bacteria (Cummings \& Macfarlane, 1991). Digestible material such as acetate, butyrate and propionate are removed from the lumen through the wall of the small bowel so indigestible material must provide the major nutrient source to the colonic microflora, alongside desquamated mucosal cells from the small bowel and small bowel secretions (Hill, 1995). Stephen \& Cummings (1980) showed that bacterial fermentation not only generated energy from carbohydrates, but also the intermediates required for protein production to support bacterial mass. It is thought that the bacterial mass in the colon is partly determined by the amount of complex carbohydrate. This would in turn ultimately determine the types of bacterial species present and may contribute to some of the symptoms present in IBS patients.

Table 2. Factors influencing fermentation in the colon (Macfarlane \& Gibson, 1995)

Chemical composition of the substrate

Amount of available substrate

Physical form of the substrate, including particle size, solubility and association with indigestible complexes such as lignins, tannins and silica

Colonic transit time

Composition of the gut microbiota with respect to species diversity and relative numbers of different types of bacteria

Ecological factors, including competitive and cooperative interaction among bacteria

Rates of depolymerisation of substrates

Substrate specificities and catabolite regulatory mechanisms of individual gut species

Fermentation strategies of individual substrate-utilising bacteria

Availability of inorganic electron receptors

$\mathrm{pH}$ of gut contents

Antibiotic therapy
Factors influencing fermentation in the colon have been summarised by Macfarlane \& Gibson (1995; Table 2). It has been shown that fermentation gases may play an important role in the aetiology of IBS. The quantity of gas in IBS patients was shown to be greater than in healthy subjects (Koide et al. 2000) while patients with IBS were shown to have impaired transit and tolerance of intestinal gas, which may in turn cause bloating (Haderstorfer et al. 1989; Serra et al. 2001).

It has been suggested that IBS may be caused by malfermentation of food residues entering the caecum from the small bowel, leading to over-production of fermentation gases, particularly hydrogen. The role of colonic malfermentation was illustrated in a study by King et al. (1998) in a controlled cross-over trial consisting of six female patients fulfilling the Rome Criteria for IBS. These and six female control subjects, both carefully matched for macronutrients and substrates for fermentation, were placed for two weeks on a standard diet followed by an exclusion diet for the same period. On the final day of each diet period, total excretion of hydrogen and methane was measured over a $24 \mathrm{~h}$ period by indirect calorimetry. After this period, patients ingested $20 \mathrm{~g}$ lactulose and breath hydrogen and methane excretion were measured over a $3 \mathrm{~h}$ fasting period. On the standard diet, IBS patients had a significantly higher maximum rate of gas production although total gas production was not greater than in controls. Following the exclusion diet, the maximum rate of gas production and hydrogen production fell in IBS patients and coincided with a significant improvement in symptoms. The authors postulated that this may be associated with alterations in fermentation activities of hydrogen-utilising bacteria and that fermentation may be of importance in the pathogenesis of IBS.

It is thought that malfermentation in IBS may be linked inextricably with food intolerances, which are a feature in a subgroup of patients with IBS. The term 'food intolerance' can be defined as a non-immunologically mediated adverse reaction to food, which can be resolved following dietary elimination and reproduced by food challenge (Zar et al. 2001). Generally, patients who suffer from food intolerance and/or colonic malfermentation present with abdominal pain, excess flatus and diarrhoea (Hunter \& Alun Jones, 1985). The potential role of food in the aetiology of IBS is illustrated in Fig. 1. Alun Jones et al. (1982) found that, in a study of twenty-one patients, specific foods were found to provoke symptoms in fourteen patients. However, no changes were found in levels of plasma glucose, histamine, immune complexes, haematocrit, eosinophil count or breath hydrogen excretion produced after either control or symptom-provoking foods. As there are no raised serum levels of immunoglobulin E, it is unlikely that patients with food-intolerant IBS suffer from classical immunologically mediated food allergies. Nanda et al. (1989) invited 200 patients with IBS to take part in an exclusion diet for three weeks. Of the 189 who completed the study, ninety-one $(48.2 \%)$ showed symptomatic improvement and $50 \%$ of these identified two to five foods that induced symptoms.

One hundred and twenty-nine patients given an exclusion diet for two weeks led to an improvement in $41 \%$ 


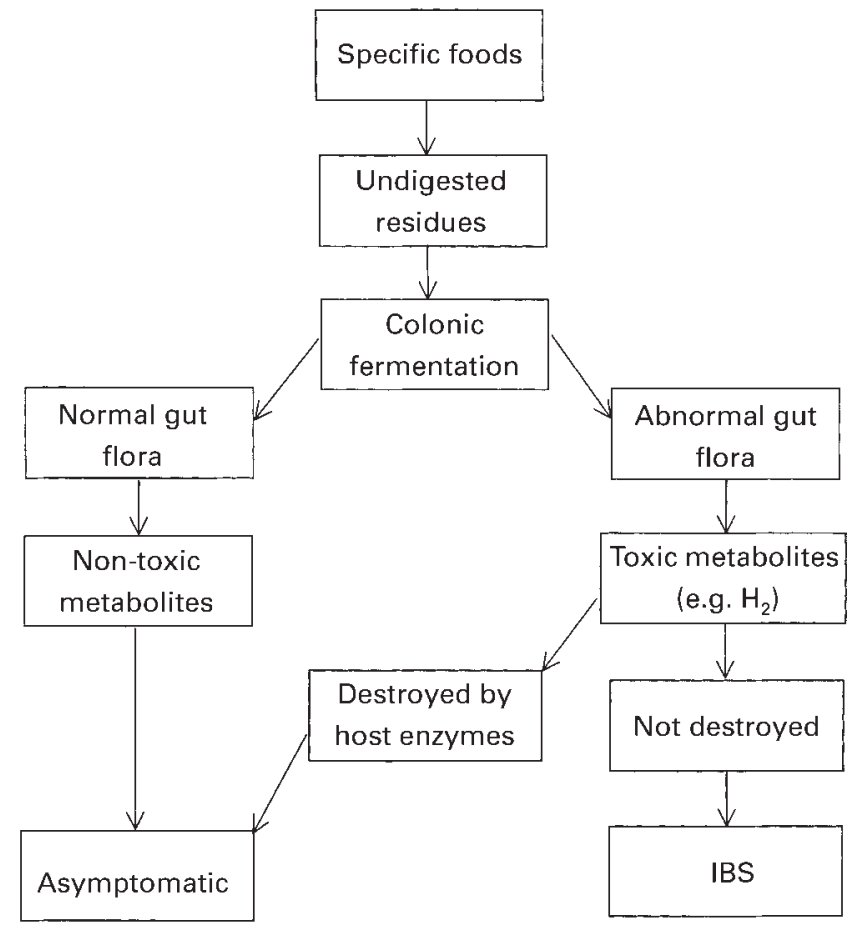

Fig. 1. The potential role of food in the aetiology of IBS.

of the patients, though subsequent identification of more uncommon food intolerances in $22 \%$ patients resulted in an overall success rate of $63 \%$ (Parker et al. 1995). Six hundred and forty-three subjects responded to a questionnaire relating food to IBS symptoms. Of these, $25 \%$ reported having sensitivity to foods (OR 2.35; $95 \%$ CI $0.41,3.93$ ), with just $3 \%$ of these reporting a swelling of the lips, or a rash. The authors suggested that these food sensitivities were a consequence of the IBS, rather than its cause (Locke et al. 2000). However, the objective changes demonstrated in gas production, prostaglandins and cytokines in other reports after food challenges in IBS patients make this unlikely (Alun Jones et al. 1982; King et al. 1998; Jacobsen et al. 2000).

\section{The role of probiotics in irritable bowel syndrome}

Probiotics are living micro-organisms that, upon ingestion in certain numbers, exert health benefits beyond basic inherent nutrition (Guarner \& Schaafsma, 1998). There have been few studies involving probiotics and IBS. This may be because IBS is a multi-factorial condition making it difficult to study homogeneous groups of patients. Halpern et al. (1996) carried out a randomised, doubleblind cross-over trial involving eighteen patients in the treatment of IBS using Lacteol Fort $\AA$, an anti-diarrhoel drug containing $5 \times 10^{10}$ heat-killed organisms/capsule of Lactobacillus acidophilus, or a placebo. Each patient received a 6-week treatment of Lacteol Fort ${ }^{\circledR}$ or placebo and then, following a 2-week washout period, a further 6-week period with either placebo or Lacteol Fort ${ }^{\circledR}$. They demonstrated a statistically significant difference $(P=0.018)$ in overall GI function, defined by clinical criteria, in the Lacteol group in comparison to those receiving placebo.

Hunter et al. (1996) administered $10^{10} \mathrm{cfu} / \mathrm{d}$ of Enterococcus faecium PR88 to twenty-eight patients with high volume diarrhoea caused by food intolerance for twelve weeks. The probiotic organism was identified in the stools of all subjects at $10^{8} / \mathrm{g}$. An increase in levels of PR88 corresponded with a decrease in excretion of Streptococcus faecalis, which ceased when PR88 feeding was stopped. There was also a symptomatic improvement in nineteen of the twenty-eight patients and a significant decrease in faecal weight. PR88 was undetectable in the faeces of all subjects within two weeks of cessation of supplementation. There were no alterations in the faecal microflora and normal biochemical and haematological parameters throughout the study. Although this was a successful study, the lack of controls means that it must be interpreted with caution.

Several trials of probiotics in IBS have used Lactobacillus plantarum $299 \mathrm{v}$ as the main probiotic organism, with varying results. Niedzielin et al. (1998) administrated a solution of L. plantarum $299 \mathrm{v}$ to IBS patients in four forms: on its own, with either trimebutin or merbeverine (two drugs frequently used in the treatment of IBS), or in solution in a pasteurised form. They found that supplementation of the probiotic in active form, with or without the drugs, produced a greater improvement of symptoms than administration of the inactivated probiotic, or of the drugs alone. Nobaeck et al. (2000) studied sixty patients with IBS who were administered a rose-hip drink containing $2 \times 10^{10} \mathrm{cfu}$ L. plantarum (DSM 9843), or a placebo drink similar in taste and colour for four weeks. This strain was the same as that used in the previously mentioned study. Over $40 \%$ of patients in the study group reported a less than $50 \%$ reduction in flatulence, compared with $18 \%$ in the placebo. However, gut function in this study was based entirely on subjective assessments. Furthermore, although flatus was reduced in the test group, it also fell significantly in the controls suggesting an overall placebo effect. There was no difference between the two groups regarding stomach bloating.

Sen et al. (2001) investigated the role of L. plantarum $299 \mathrm{v}$ on symptoms and colonic fermentation in twelve patients with IBS, in a double-blind, controlled, crossover 4-week trial. Patients received $6.25 \times 10^{9} \mathrm{cfu} / \mathrm{d} L$. plantatrum $299 \mathrm{v}$, or a placebo drink similar in taste and colour. Fermentation was assessed by indirect calorimetry over a $24 \mathrm{~h}$ period, after which breath hydrogen was measured for $3 \mathrm{~h}$ after ingestion of $20 \mathrm{ml}$ of lactulose. Although there was a significant decrease in breath hydrogen levels in the probiotic group at 120 min after ingestion of lactulose $(P=0.019)$, there was no decrease in total hydrogen production, or any symptomatic improvement.

The role of Lactobacillus casei strain GG (LGG) in IBS was studied in a randomised, double-blind cross-over trial. Twenty-four patients fulfilling the Rome Criteria for IBS were entered into the study and randomised to receive either $1 \times 10^{10} \mathrm{cfu} / \mathrm{d}$ enterocoated LGG or a placebo. Nineteen patients completed the trial and there was no significant difference in pain, urgency or bloating between the two groups, though there was a reduction in diarrhoea in 
the LGG group. The authors concluded that LGG alone did not have an effect on symptoms of IBS, though further work may be warranted in the subgroup of patients that are diarrhoea-predominant (O'Sullivan \& O'Morain, 2000).

\section{Conclusion}

The evidence for the use of probiotic bacteria in IBS is so far inconclusive - the trials that have been performed have centred on a symptomatic reduction or cure and have produced varying results. Currently, no organism can be confidently recommended to patients as being likely to help their symptoms. However, the abnormalities seen in the colonic flora of IBS suggest that a probiotic approach will ultimately be justified. It may be that the future use of probiotics will lie in the prevention of damage to the intestinal microflora following antibiotics or gastroenteritis, which in turn may prevent the onset of symptoms associated with IBS.

\section{References}

Alun Jones V, McLaughlin P, Shorthouse M, Workman E \& Hunter JO (1982) Food intolerance: a major factor in the pathogenesis of irritable bowel syndrome. Lancet 2, 1115-1117.

Alun Jones V, Wilson AJ, Hunter JO \& Robinson RE (1984) The aetiological role of antibiotic prophylaxis with hysterectomy in irritable bowel syndrome. Journal of Obstetrics and Gynaecology 5, Suppl. 1, S22-S23.

Balsari A, Ceccarelli A, Dubini F, Fesce E \& Poli G (1982) The fecal microbial population in the irritable bowel syndrome. Microbiologica 5, 185-194.

Bradley HK, Wyatt GM, Bayliss CE \& Hunter JO (1987) Instability in the faecal flora of a patient suffering from food-related irritable bowel syndrome. Journal of Medical Microbiology 23, 29-32.

Camilleri M (2001) Management of the irritable bowel syndrome. Gastroenterology 120, 652-668.

Cummings JH \& Macfarlane GT (1991) The control and consequences of bacterial fermentation in the colon. Journal of Applied Bacteriology 70, 443-459.

Farthing MJG (1995) Irritable bowel, irritable body, or irritable brain? British Medical Journal 310, 171-175.

Finegold SM, Atterbury HR \& Sutter SL (1983) Normal indigenous intestinal flora. In Human Intestinal Microflora in Health and Disease, pp. 3-31 [D Hentges, editor]. London: Academic Press.

Guarner F \& Schaafsma GJ (1998) Probiotics. International Journal of Food Microbiology 39, 237-238.

Gwee KA (2001) Postinfectious irritable bowel syndrome. Current Treatment Options in Gastroenterology 4, 287-291.

Gwee KA, Graham JC, McKendrick MW, Collins SM, Marshall JS, Walters SJ \& Read NW (1996) Psychometric scores and persistence of irritable bowel after infectious diarrhoea. Lancet 347, 617-618.

Haderstorfer B, Psycholgin D, Whitehead WE \& Schuster MM (1989) Intestinal gas production from bacterial fermentation of undigested carbohydrate in irritable bowel syndrome. American Journal of Gastroenterology 84, 375-378.

Halpern GM, Prindiville T, Blankenburg M, Hsia T \& Gershwin ME (1996) Treatment of irritable bowel syndrome with Lacteol
Fort: a randomized, double-blind, cross-over trial. American Journal of Gastroenterology 91, 1579-1585.

Hill MJ (1995) The normal gut bacterial flora. In Role of Gut Bacteria in Human Toxicology and Pharmacology, pp. 3-19 [MJ Hill, editor]. London: Taylor \& Francis.

Hooker KD \& dePiro JT (1988) Effect of antimicrobial therapy on bowel flora. Clinical Pharmacology 7, 878-888.

Hunter JO (1991) Food allergy — or enterometabolic disorder? Lancet 338, 495-496.

Hunter JO \& Alun Jones V (1985) Studies on the pathogenesis of irritable bowel syndrome produced by food intolerance. In Irritable Bowel Syndrome, pp. 185-190 [NW Read, editor]. London: Grune \& Stratton Ltd.

Hunter JO, Lee AJ, King TS, Barratt MEJ, Linggood MA \& Blades JA (1996) Enterococcus faecium strain PR88 - an effective probiotic. Gut 38, Suppl. 1, A62.

Jacobsen MB, Aukrust P, Kittang E, Müller F, Ueland T, Bratlie J, Bjerkeli V \& Vatn MH (2000) Relation between food provocation and systemic immune activation in patients with food intolerance. Lancet 356, 400-401.

King TS, Elia M \& Hunter JO (1998) Abnormal colonic fermentation in irritable bowel syndrome. Lancet 352, 1187-1189.

Koide A, Yamaguchi T, Odaka T, Koyama H, Tsuyuguchi T, Kitahara H, Ohto M \& Saisho H (2000) Quantitative analysis of bowel gas using plain abdominal radiograph in patients with irritable bowel syndrome. American Journal of Gastroenterology 95, 1735-1741.

Lidbeck A \& Nord CE (1993) Lactobacilli and the normal human anaerobic microflora. Clinical Infectious Diseases 16, S181S187.

Lidbeck A \& Nord CE (1994) Lactobacilli, normal human microflora and antimicrobial treatment. In Human Health: The Contribution of Microorganisms, pp. 95-110 [SAW Gibson, editor]. London: Springer-Verlag Limited.

Locke GR, Zinsmeister AR, Talley NT, Fett SL \& Melton J (2000) Risk factors for irritable bowel syndrome: role of analgesics and food sensitivities. American Journal of Gastroenterology 95, 157-165.

Macfarlane GT \& Gibson GR (1995) Microbiological aspects of short chain fatty acid production in the large bowel. In Physiological and Clinical Aspects of Short Chain Fatty Acid Metabolism, pp. 87-105 [JH Cummings, JL Rombeau and T Sakata, editors]. Cambridge: Cambridge University Press.

Madden JAJ, Plummer S, Sen S, Dear K, Tarry S \& Hunter JO (2001) Comparison of the caecal and faecal microflora of healthy subjects and patients with irritable bowel syndrome (IBS). Gut 48, Suppl. 1, A58.

Marshall JC (1999) Gastrointestinal flora and its alterations in critical illness. Current Opinions in Nutrition and Metabolic Care 2, 405-411.

Maxwell PR, Mendall MA \& Kumar D (1997) Irritable bowel syndrome. Lancet 350, 1691-1695.

Mendall MA \& Kumar D (1998) Antibiotic use, childhood affluence and irritable bowel syndrome. European Journal of Gastroenterology and Hepatology 10, 59-62.

Nanda R, James R, Smith H, Dudley CR \& Jewell DP (1989) Food intolerance and the irritable bowel syndrome. Gut 30, 1099-1104.

Neal KR, Hebden J \& Spiller R (1997) Prevalence of gastrointestinal symptoms six months after bacterial gastroenteritis and risk factors for the development of the irritable bowel syndrome: postal survey of patients. British Medical Journal 314, 779-782.

Neilson OH, Jorgenson S, Pederson K \& Justensen T (1994) Microbiological evaluation of jejunal aspirates and faecal samples after oral administration of bifidobacteria and lactic acid bacteria. Journal of Applied Bacteriology 76, 469-474. 
Niedzielin K, Kordecki H \& Kosik R (1998) New possibility in the treatment of irritable bowel syndrome: probiotics as a modification of the microflora of the colon. Gastroenterology 114, A402.

Nobaek S, Johansson ML, Molin G, Ahrne S \& Jeppsson B (2000) Alteration of intestinal microflora is associated with reduction in abdominal bloating and pain in patients with irritable bowel syndrome. American Journal of Gastroenterology 95, $1231-1238$.

O'Sullivan MA \& O'Morain CA (2000) Bacterial supplementation in the irritable bowel syndrome. A randomised double-blind placebo-controlled crossover study. Digestive Diseases and Science 32, 302-304.

Parker TJ, Naylor SJ, Riordan AM \& Hunter JO (1995) Management of patients with food intolerance in irritable bowel syndrome: the development and use of an exclusion diet. Journal of Nutrition and Dietetics 8, 159-166.

Pimental M, Chow EJ \& Lin HC (2000) Eradication of small intestinal bacterial overgrowth reduces symptoms of irritable bowel syndrome. American Journal of Gastroenterology 95, 3503-3506.

Rodríguez LAG \& Ruigómez A (1999) Increased risk of irritable bowel syndrome after bacterial gastroenteritis: cohort study. British Medical Journal 318, 565-566.

Savage DC (1977) Microbial ecology of the gastrointestinal tract. Annual Reviews in Microbiology 31, 107-133.

Sen S, Mullan M, Parker TJ, Woolner J, Tarry SA \& Hunter JO (2001) Effects of Lactobacillus plantarum 299v on symptoms and colonic fermentation in irritable bowel syndrome (IBS). Gut 48, Suppl. 1, A57.

Serra J, Azpiroz F \& Malagelada J-R (2001) Impaired transit and tolerance of intestinal gas in the irritable bowel syndrome. Gut 48, 14-19.

Stephen AM \& Cummings JH (1980) Mechanism of action of dietary fibre in the human colon. Nature 284, 5753, 283-284.

Thompson WG, Longstreth GF, Drossman DA, Heaton KW, Irvine EJ \& Muller-Lissner SA (1999) Functional bowel disorders and functional gastrointestinal disorders. Gut $\mathbf{4 5}$, $1143-1147$

Van der Waiij D (1983) Antibiotic Choice: The Importance of Colonisation Resistance. Chichester: Research Studies Press.

Witsell DL, Garrett CG, Yarbrough WG, Dorrestein SP, Drake AF \& Weissler MC (1995) Effect of Lactobacillus acidophilus on antibiotic-associated gastrointestinal morbidity: a prospective randomized trial. Journal of Otolaryngology 24, 230-233.

Wyatt GM, Bayliss CE, Lakey AF, Bradley HK, Hunter JO \& Alun Jones V (1988) The faecal flora of two patients with food-related irritable bowel syndrome during challenge with symptom-provoking foods. Journal of Medical Microbiology 26, 295-299.

Zar S, Kumar D \& Benson MJ (2001) Food hypersensitivity and irritable bowel syndrome. Alimentary Pharmacology and Therapeutics 15, 439-449. 